\title{
Factores de riesgo y perfil epidemiológico de la cisticercosis porcina/teniasis humana en el distrito de Zapatero
}

\section{Risk factors and epidemiological profile of swine cysticer- cosis/ human taeniasis in Zapatero District}

Sánchez-Cárdenas, Hugo 1[0000-0003-1560-2402]; Puicón, Victor ${ }^{1[0000-0003-2532-}$ 2551] y Arévalo-Ramírez, Heriberto ${ }^{1[0000-0002-3252-6301]}$

${ }^{1}$ Universidad Nacional de San Martín, Tarapoto, Perú hsanchez@unsm.edu.pe

Resumen. El presente estudio tuvo como objetivo evaluar los factores de riesgo y el perfil epidemiológico de la cisticercosis porcina/teniasis humana en el Centro Poblado Menor de Bagazán. Se trabajó con una muestra de 100 personas encuestadas, 179 muestras coprológicas humanas y un análisis cualitativo y cuantitativo de 176 cerdos. Los resultados demostraron que el $61 \%$ de los pobladores encuestados mencionaron que los factores sociopolíticos son buenos y el 39\% manifestaron que es regular, en cuanto a los factores económicos el $100 \%$ de los pobladores manifiestan que las condiciones económicas son malas. El 64\% de los criadores de cerdos mencionan que crían los cerdos sueltos encerrados en un corral, el $24 \%$ crían sueltos sin control, el $6 \%$ lo sueltan por horas y el $4 \%$ crían a los cerdos amarrados. Para el sistema de faenamiento el $93 \%$ de los encuestados manifiestan que es regular, el $5 \%$ consideran que es bueno y el $2 \%$ que es malo, encontrándose diferencias significativas con $p=0,00$. De los 42 cerdos sacrificados 3 cerdos estaban infestados con cisticercos en el músculo del lomo la cara, pero era una infestación leve. El 100\% de las personas en las que se realizó análisis coparasitológico mediante la técnica cuantitativa de Mc Master modificado y técnica cualitativa de flotación en el laboratorio para detectar teniasis en humanos dieron como resultado negativo.

Palabras clave: cisticercosis porcina, factores de riesgo, perfil epidemiológico, teniasis humana

Citar como: SánchezCárdenas, H., Puicón, V. \& Arévalo-Ramírez, $\mathrm{H}$. (2021). Factores de riesgo y perfil epidemiológico de la cisticercosis porcina/teniasis humana en el distrito de Zapatero. Revista de Veterinaria y Zootecnia Amazónica, 1(1), 31-42. https://doi.org/10.51252/re vza.v1i1.140

Recibido: $14 / 04 / 2021$

Aceptado: 21/06/2021

Publicado: 28/07/2021

\begin{abstract}
The objective of this study was to evaluate the risk factors and the epidemiological profile of porcine cysticercosis / human taeniasis in the Centro Poblado Menor de Bagazán. We worked with a sample of 100 people surveyed, 179 human stool samples and a qualitative and quantitative analysis of 176 pigs. The results showed that $61 \%$ of the surveyed residents mentioned that the socio-political factors are good and $39 \%$ stated that it is regular, as for the economic factors, $100 \%$ of the residents stated that the economic conditions are bad. $64 \%$ of pig farmers mention that they raise loose pigs locked in a pen, $24 \%$ raise loose uncontrolled, $6 \%$ release it for hours and $4 \%$ raise tied pigs. For the slaughter system, 93\% of those surveyed state that it is regular, 5\% consider that it is good and $2 \%$ that it is bad, finding significant differences with $\mathrm{p}=0.00$. Of the 42 pigs slaughtered, 3 pigs were infested with cysticerci in the back muscle of the face, but it was a mild infestation. $100 \%$ of the people in whom a coparasitological analysis was performed using the modified McMaster quantitative technique and qualitative flotation technique in the laboratory to detect taeniasis in humans gave negative results.
\end{abstract}

Keywords: epidemiological profile, human teniasis, risk factores, swine cisticercosis 


\section{$1 \quad$ Introducción}

Los términos cisticercosis y teniasis se refieren a infecciones zoonóticas transmitidas por los alimentos con formas larvales y adultas de Taenia solium, respectivamente. Las características importantes de estas zoonosis es que las larvas son transmitidas por la carne y la etapa adulta se desarrolla solo en el intestino del huésped humano (1). La cisticercosis muscular y cerebral (en el humano y en el cerdo), cuya modalidad más dramática es la neurocisticercosis humana, es provocado por la forma larvaria (Cisticercosis celulosae) que proviene de la forma adulta Taenia solium, que vive en el intestino delgado del humano infectado.

La Organización Mundial de la Salud (OMS), reportó que más del 80\% de 50 millones de personas que padecen epilepsia a nivel mundial, habitan en países subdesarrollados. La larva Taenia solium es la causante del 30\% de los casos de epilepsia localizada en diversas zonas endémicas donde las personas realizan sus actividades en convivencia con cerdos (2-5). En la población China, cerca de 1,26 millones de personas son portadores de tenia, además tres millones de humanos padecen cisticercosis (2).

En el Perú, los habitantes de zonas alto andinas son las más afectadas con la presencia de cisticercosis/teniasis y en menor medida los pobladores de la costa y selva. En las zonas rurales la prevalencia había alcanzado un $17 \%$ y $75 \%$ de teniasis y cisticercosis en humanos y porcinos, respectivamente (3). La pobreza, la falta de educación sanitaria de los pobladores, deficiencias en el manejo de eliminación de excretas y residuos sólidos al aire libre; el consumo de agua no potabilizada, la crianza y beneficio de cerdos sin revisión ni autorización sanitaria, y el consumo de esta carne; son factores que favorecen la diseminación de cisticercosis/teniasis (1). En Tarapoto se encontraron incidencias que han variado de $43 \%$ en Maceda (4) a $49 \%$ en Churusapa (5).

En la epidemiología o difusión, también se dispone de gran información estadística de prevalencias, incidencias, casuísticas, etc. pero, poco o nada se ha estudiado sobre la sistematización de las variables o factores profundamente ligados con el comportamiento humano, especialmente: Educación, creencias, hábitos, costumbres y niveles socioeconómicos de las comunidades de influencia. La incidencia de la cisticercosis aumenta debido a la falta de tratamiento de las aguas residuales urbanas, que contaminan las fuentes de agua que abastecerán a los animales e incluso al hombre mismo. La falta de pozos en las áreas rurales contribuye a la contaminación ambiental, con casos en que los animales terminan consumiendo heces humanas. El riego con agua contaminada de jardines y huertos es una fuente importante de infección para los seres humanos. 
En los camales, la fiscalización de animales parasitados es prácticamente inexistente, dado que actualmente hay libertad de camaleo o beneficiamiento de los cerdos, a tal punto que en los camales distritales solo se procesan bovinos. En cuanto a la normatividad para el control y prevención, inexiste un marco legal como Ley, Decreto Supremo o Legislativo, y lo que pueda haber son aisladas, ordenanzas municipales e inquietudes institucionales. Por otro lado, se ha dejado la capacitación y la transferencia tecnológica, a la iniciativa del sector privado, a tenor de la Normatividad del SENASA.

La teniasis y la cisticercosis prevalecen en áreas urbanas y rurales, donde a las prácticas tradicionales, sanitarias e higiénicas se asocian a la ignorancia y pobreza. El conocimiento del ciclo de vida de la Taenia solium es esencial para entender mejor la enfermedad. El control de estas enfermedades tiene como estrategia fundamental, la interrupción del ciclo vital del parásito, evitando la infección de animales y seres humanos, a través del control higiénicosanitario, como la construcción de sistemas de alcantarillado, la concienciación de la población sobre prácticas de higiene, tales como el no consumo de carne cruda y la higiene adecuada de los vegetales a consumir crudos; mejora de las condiciones de reproducción, como los cerdos, impidiendo el acceso de los animales a las heces humanas; y promover la inspección estricta de los productos cárnicos evitando el sacrificio y el comercio clandestino (6).

Por lo antes mencionado se realizó esta investigación con el propósito de evaluar los factores de riesgo y el perfil epidemiológico de la cisticercosis porcina/teniasis humana en el Centro Poblado Menor de Bagazán del Distrito de Zapatero en el año 2019. Los resultados permitirán a las autoridades y población en general conocer su situación real respecto a este problema de salud pública y tomar acciones para su control.

\section{Materiales y Métodos}

Se ha llevado a cabo una investigación aplicada porque se recomendó estrategias o mecanismos que permitan la reducción o eliminación de cisticercosis porcina y teniasis humana, bajo un nivel descriptivo ya que se describió los factores de riesgo y perfil epidemiológico de la cisticercosis porcina/teniasis humana en las condiciones de vida del Centro Poblado Menor de Bagazán y en base a los resultados encontrados se planteó un protocolo que permita disminuir o eliminar la presencia de cisticercosis.

La unidad muestral estuvo conformada por 176 porcinos que fueron analizados de manera cuantitativa y cualitativa la presencia de cisticercosis en porcinos. También se realizó el análisis cuantitativo y cualitativo de las heces de 179 personas que crían o consumen cerdos en el Centro Poblado Menor de Bagazán. 
Para la obtención de datos primarios se elaboró una encuesta de 31 preguntas. Los ítems fueron valorados en una escala tipo Likert distribuida en dimensiones sociopolíticas, económicas, crianza del porcino y faenamiento del porcino. Además, se empleó una ficha de monitoreo de cisticercosis en porcinos para su identificación, determinando la raza, la edad y la categoría, así también se hizo la observación macroscópica de la lengua de los cerdos vivos y sacrificados para determinar cisticercosis.

Se contó también con la elaboración de una ficha de toma de muestras (coprológicos y de resultados) y monitoreo de teniasis en humanos para los análisis coprológicos de las personas criadoras y consumidores de carne de porcino que determinase la presencia de teniasis humana. Se suma el faenamiento para el proceso de matanza de los cerdos, el cual consiste en quitarle la vida, el pelado con agua caliente, el lavado exterior del animal, el eviscerado y los cortes de la carne para su comercialización.

El procesamiento de datos consistió en ordenar, depurar, homogenizar, estandarizar, codificar, tabular y elaborar la base de datos, para comprobar las hipótesis planteadas, utilizando el programa estadístico Statistical Package for the Social Sciences (SPSS) v.22.

\section{Resultados y discusiones}

\subsection{Factores sociopolíticos y económicos que se asocian a la presencia de Taenia solium}

Las acciones que realizan las autoridades representantes del gobierno en diferentes aspectos como la prevención en salud pública a través de los centros de salud y las mejoras sociales en la comunidad a través de las municipalidades, son percibidas y expresadas por los pobladores. En la tabla 1 se muestran la calificación de los pobladores a los factores sociales y políticos en el Centro Poblado Menor de Bagazán.

Tabla 1.

Distribución de la población según servicios básicos (Factores sociopolíticos), Bagazán 2019

\begin{tabular}{lrr}
\hline $\begin{array}{l}\text { Factor socieconómico / } \\
\text { Servicio básico }\end{array}$ & \multicolumn{1}{c}{ Frecuencia } & \multicolumn{1}{c}{ Porcentaje } \\
\hline Regular & 39 & $39 \%$ \\
Bueno & 61 & $61 \%$ \\
Total & $\mathbf{1 0 0}$ & $\mathbf{1 0 0 \%}$ \\
\hline
\end{tabular}

El factor económico de la población es fundamental para luchar contra las enfermedades, puesto que influyen en sus actividades con mejores sistemas de producción evitando riesgos. En la tabla 2 se observa la percepción de los habitantes del Centro Poblado Menor de Bagazán respecto a la situación económica. 
Tabla 2.

Distribución porcentual de la crianza de cerdos, según factor económico, Bagazán 2019

\begin{tabular}{lrr}
\hline \multicolumn{1}{c}{ Factor económico } & Frecuencia & Porcentaje \\
\hline Malo & 100 & $100 \%$ \\
Bueno & 0 & 0 \\
Total & $\mathbf{1 0 0}$ & $\mathbf{1 0 0 \%}$ \\
\hline
\end{tabular}

\subsection{Análisis del sistema de crianza y faenamiento de porcinos}

Existen diversas formas de crianza de cerdos, unas más tradicionales que otros, la crianza de cerdos siempre sueltos y sin ningún control son focos de contaminación de las fuentes de agua siendo un riesgo para la salud de los habitantes que conviven con los animales. En la tabla 3 se muestra los sistemas de crianza de los cerdos en el Centro Poblado Menor de Bagazán.

Tabla 3.

Distribución porcentual de la crianza de cerdos según sistema de explotación, Bagazán 2019

\begin{tabular}{lrr}
\hline Sistema de explotación & Frecuencia & Porcentaje \\
\hline Siempre sueltos & 24 & $24 \%$ \\
Sueltos por horas & 6 & $6 \%$ \\
Sueltos en un corral & 64 & $64 \%$ \\
Amarrados & 4 & $4 \%$ \\
Otra forma & 2 & $2 \%$ \\
Total & $\mathbf{1 0 0}$ & $\mathbf{1 0 0 \%}$ \\
\hline
\end{tabular}

El beneficiado o sistema de faenamiento de los cerdos es muy importante que se realice siguiendo buenas prácticas, porque al eliminar desechos como heces, pelos, sangres y otros se puede contaminar el medio ambiente, a otros animales y aun a las personas de entorno. En la tabla 4 se muestra la calificación de los pobladores respecto al sistema de faenamiento de cerdos en el Centro Poblado Menor de Bagazán.

Tabla 4.

Distribución porcentual del sistema de faenamiento, Bagazán 2019

\begin{tabular}{lrr}
\hline Sistema de faenamiento & Frecuencia & Porcentaje \\
\hline Malo & 2 & $2 \%$ \\
Regular & 93 & $93 \%$ \\
Bueno & 5 & $5 \%$ \\
Total & $\mathbf{1 0 0}$ & $\mathbf{1 0 0 \%}$ \\
\hline
\end{tabular}

\subsection{Nivel de incidencia de cerdos portadores de Cisticercos Celulosae}

Los cerdos portadores de Cisticercos Celulosae son los principales focos de contaminación para otros cerdos y las personas que habitan en el entorno que pueden ser contaminados a través de las fuentes de agua o alimento con huevos de cisticercosis. En la tabla 5 se presenta la incidencia encontrada en cerdos sacrificados en el Centro Poblado Menor de Bagazán. 
Tabla 5.

Incidencia de cerdos portadores de Cisticercos Celulosae

\begin{tabular}{lrr}
\hline \multicolumn{1}{c}{ Nivel } & Frecuencia & Porcentaje \\
\hline Positivo & 3 & $7 \%$ \\
Negativo & 39 & $93 \%$ \\
Total & $\mathbf{4 2}$ & $\mathbf{1 0 0 \%}$ \\
\hline
\end{tabular}

Al realizar el análisis de significancia se encontró que existe diferencia estadísticamente significativa $(\rho<0.05)$ con valor de $\rho=00$ entre la incidencia de cerdos positivos y negativos. La incidencia de los cerdos se evaluó en seis meses de estudio en el Centro Poblado Menor de Bagazán.

\subsection{Prevalencia de teniasis en humanos}

Los seres humanos pueden infectarse con tenia al consumir carne de cerdo infestado con cisticercos, si la carne tiene una cocción insuficiente el riesgo es mayor.

Tabla 6.

Teniasis en humanos

\begin{tabular}{lrr}
\hline \multicolumn{1}{c}{ Nivel } & Frecuencia & Porcentaje \\
\hline Positivo & 0 & 0 \\
Negativo & 179 & $100 \%$ \\
Total & $\mathbf{1 7 9}$ & $\mathbf{1 0 0 \%}$ \\
\hline
\end{tabular}

En la tabla 6, se observa que el $100 \%$ de las personas en las que se realizó análisis coparasitológico por la técnica cuantitativa de Mc Master modificado y la técnica cualitativa de flotación para detectar teniasis en humanos dieron como resultado negativo.

\subsection{Propuesta de un protocolo de prevención y control de la cisticercosis porcina y teniasis humana}

\section{a) Crianza}

Corral cerrado: para evitar la contaminación del agua y otras fuentes importantes que puedan poner en riesgo la salud pública, y considerando que los habitantes del Centro Poblado Menor de Bagazán tienen suficientes áreas disponibles, los que se dedican a criar cerdos deben construir corrales para utilizando los materiales que se cuenten en la zona y el tamaño del corral debe ser según la cantidad de cerdos que se deseen criar.

Antes de la construcción del corral se debe verificar que el terreno tenga buen drenaje y sea permeable para evitar la formación de lodos que perjudiquen al animal. Los corrales deben contener un local donde el animal pueda dormir y protegerse de la lluvia o el excesivo calor, la construcción de estos locales debe ser teniendo en cuenta la dirección de la luz solar y los vientos dominantes. 
Cerdos sueltos: en los corrales se encontró los cerdos sueltos, esto reduce el estrés en comparación con los cerdos amarrados, esto le permite tener una mejor alimentación porque pueden consumir pasto, agua y alimento según sus necesidades fisiológicas y en consecuencia se tendrá animales más sanos.

Revisión periódica: cada seis meses se realizó una revisión completa del estado de salud de los cerdos, con el fin de controlar cualquier tipo de enfermedad que los esté afectando. Los propietarios deben conocer las técnicas de reconocimiento de cisticercosis con el propósito de que sea controlado antes que los cistercos se diseminen fuera del corral.

\section{b) Alimentación}

Alimentos limpios: todos los alimentos que se den a los cerdos deben ser colocados en un depósito limpio y libre de contaminantes, se debe tener en cuenta la fuente de obtención de los alimentos para evitar contaminación cruzada. Es decir, cuidar que los alimentos consistentes en restos de comida que sobraron de las personas no tengan pedazos de carne de cerdo, sangre u otros que estén contaminados con cisticercos.

Comederos adecuados: los comederos de los animales deben ser de material resistente, que se puedan lavar con facilidad, deben ser de tamaño adecuado para que los animales no ingresen ni ensucien sus alimentos. Estos comederos deben estar ubicados en un extremo del corral de tal manera que los propietarios no tengan la necesidad de ingresar al corral colocar el alimento y solo se pueda hacer desde la parte externa, evitando estar en contacto directo con los animales.

Agua limpia: los cerdos deben tener disponibilidad agua limpia para que beban ad libitum (un cerdo requiere aproximadamente 2 litros de agua/día con $20 \mathrm{~kg}$ de peso), el mismo que debe estar colocado en depósitos adecuados lavados diarios y el agua debe ser cambiado diariamente para evitar la propagación de zancudos.

\section{c) Faenamiento}

Antes del faenamiento los cerdos deben pasar por inspección sanitaria por la autoridad designada en el sector.

El proceso de faenamiento se inicia con el degüello y sangrado del animal, siendo fundamental tener control en el recojo y destino de la sangre, porque se debe evitar que ésta fluya a la tierra o sea consumido crudo por otros animales. Luego en el proceso de pelado y chamuscado se debe incinerar los restos de pelo y pezuñas. El agua con la que se lava el cuerpo del animal debe ser recibida en depósitos y desechados adecuadamente, de la misma manera las heces y otros desperdicios deben ser eliminados tratando de no contaminar el medio ambiente. 
Buena limpieza: durante el faenamiento la carne debe ser cuidadosamente lavada y separada de las vísceras y restos que puedan contaminar, se debe enjuagar con abundante agua y colgar en un lugar limpio y protegido de las moscas.

Verificación visual: realizando cortes en diferentes partes del cerdo lavado se debe hacer revisión visual de la posible presencia de cisticercos.

\section{d) Consumo}

Carne sana: los habitantes del Centro Poblado Menor de Bagazán en un 100\%, conocen y deben continuar practicando el hábito de consumir carne totalmente sana y libre de cisticercos, con el propósito de evitar la teniasis humana y la propagación de cisticercosis en el entono en que habitan.

Buena limpieza: antes de preparar alimentos con carne de cerdo, se debe revisar que no contenga cisticercos y se debe hacer una limpieza profunda, con la finalidad de asegurar su calidad microbiológica.

Cocción completa: la carne de cerdo antes de su consumo debe ser bien cocinada, freída o asada; verificando su cocción completa.

Los habitantes de la comunidad del Centro Poblado Menor de Bagazán tienen como principal fuente de ingreso la producción agrícola de plátanos, la cría de cerdos como medio de subsistencia. Crían cerdos criollos por ser poco exigente en calidad de insumos proteicos, son alimentados con plátano, yuca, algunas veces polvillo. El 100\% de la población se dedica a la agricultura con un ingreso promedio de 40 soles diario.

Al respecto (7) mencionan que el predominio de un bajo ingreso familiar en las comunidades encuestadas que tienen ingresos menores a un salario mínimo mostraron mayor evidencia positiva de cisticercosis bovina y porcina, acompañado por las respectivas implicaciones de la deficiencia sanitaria y el bajo nivel de conocimiento demostrado por la mayoría de los encuestados $(75,3 \%)$ sobre la naturaleza de la cisticercosis, son signos indicativos de la ocurrencia común de la enfermedad en las regiones encuestadas, donde casi el $100 \%$ de la población rural cultiva el hábito de consumir carne de res y cerdo (7).

Los habitantes del Centro Poblado Menor de Bagazán han mejorado y continúan mejorando sus condiciones de vida, porque reciben apoyo del municipio de la jurisdicción del distrito de Zapatero, con presupuestos vía proyectos, saneamiento básico, letrinas, agua entubada. El Ministerio de Salud trabaja salud ambiental, visitando casa por casa incentivando en la comunidad el correcto lavado de manos, buenas prácticas de manufacturas de alimentos, bebidas, comidas, etc. Como resultado las personas se organizan para ver los temas de salud pública, ambiental, trabajan organizados en la limpieza bienestar de su comunidad, velan por el 
agua, saneamiento básico letrinas, agua entubada, corrales de encierro de los cerdos ya poseen al 98\% evitando así cerdos vagabundos (sueltos) y así van disminuyendo la contaminación de las heces, o la tenía, del hombre al cerdo.

En una investigación realizada en Tumbes, (8) encontró que el nivel de incidencia de cisticercosis en cerdos en los tres primeros meses del año 2000 en el distrito de Matapalo, fue de $11,5 \pm 3,5 \%$ (36/314) siendo los de mayor incidencia la edad del cerdo demostró que los que tenían meses de nacido se infectaban a menor edad ( 5 a 8 meses), en cuanto a los caseríos se encontró mayor incidencia en aquello dónde los cerdos tenían mayor acceso al consumo de heces humanas contaminadas con huevos de Taenia solium.

En la tabla 3 reporta que el $64 \%$ de los criadores de cerdos mencionan que crían los cerdos sueltos pero encerrados en un corral, el $24 \%$ crían sueltos, el $6 \%$ lo sueltan por horas y el $4 \%$ crían a los cerdos amarrados. Al realizar el análisis de significancia se encontró que existe diferencia estadísticamente significativa entre los sistemas de crianza de los cerdos siempre suelto y sueltos en un corral. (1) menciona que los principales factores de riesgo asociados con el complejo teniasis-cisticercosis, son: La crianza de cerdos sueltos en lugares donde no hay inodoro, el comercio de carne de cerdo sin inspección sanitaria, el consumo de carne de cerdo cruda o mal cocinada y personas con teniasis involucradas en la cría de cerdos.

El sacrificio de los cerdos se realiza por corte de la vena yugular, en el proceso de faenamiento se retiran los pelos a fuego por soplete, o chamuscado con hoja de coco, luego realizan el eviscerado en batan grande, el seccionado por piezas lo hacen cuando el cerdo está colgado en el arco en ganchos. El 93\% de los encuestados manifiestan que el sistema de faenamiento en el Centro Poblado Menor de Bagazán es regular, el 5\% consideran que es bueno y el 2\% que es malo. Al realizar el análisis de significancia se encontró que existe diferencia estadísticamente significativa entre la apreciación de los pobladores del sistema de faenamiento regular y las consideraciones malas y buenas.

La sociedad del Centro Poblado Menor de Bagazán tiene entre sus costumbres el celebrar las fiestas patronales, para ello se organizan y las familias donan cerdos que serán faenados en un solo día previo a la fiesta. En la fiesta patronal del año 2019, 42 cerdos fueron faenados, encontrándose 3 con cisticercosis en el musculo del lomo y la cara, pero una infección leve. Sin embargo, al realizar análisis coparasitológico a 179 personas no se encontró teniasis en humanos. Los habitantes mencionan que están capacitados para reconocer los cistercos en la carne de cerdo evitando su consumo y la correcta eliminación de la carne contaminada.

$\mathrm{Al}$ respecto, (9) menciona que luego de inspeccionar 500 cerdos faenados en el camal municipal del Cantón-Ecuador, encontró un $(0,2 \%)$ cerdo de raza mestiza con cisticercosis en su carne; en la investigación realizada en el Camal Municipal de Yurimaguas (10) encontró que el nivel de 
incidencia de cisticercosis en cerdos fue de 0,48\% y 0,74\% en los años 2012-2013 respectivamente. La mayor infestación de cisticercosis se encontró el corazón con 85,7\% (2012) y $83,3 \%$ (2013) y la lengua con $92,9 \%$ (2012) y $83,3 \%$ (2013).

La comunidad aprendió a usar letrinas, criar los cerdos en sus corrales cerrados la gran mayoría, no hay un contacto de animales con las personas, de forma que se organizan para mejorar su calidad de vida en la salud pública y ambiental; faltando trabajar el agua que sea de óptima calidad y no agua con materias fecales.

El monitoreo de la cisticercosis porcina ha sido el método más utilizado para evaluar las actividades de control de Taenia solium. Es más fácil y barato que realizar pruebas en humanos, ya que no implica el mismo nivel de experiencia y/o consideraciones éticas. Una de las principales ventajas de monitorear la cisticercosis porcina sobre las infecciones humanas es la vida mucho más corta de los cerdos, lo que proporciona una medida sensible al tiempo. Existen varios métodos disponibles para detectar la cisticercosis porcina directa o indirectamente; sin embargo, varían mucho en su sensibilidad, especificidad y calidad de la evidencia disponible para respaldar las características de rendimiento de diagnóstico que se han reivindicado (11).

El mantenimiento del complejo de teniasis-cisticercosis está directamente relacionado con las condiciones socioeconómicas, ambientales e higiénico-culturales de la población, así como con los estándares de cría de animales, sistemas de inspección sanitaria y de sacrificio y consumo de carne (12).

\section{Conclusiones}

No se determinó asociación significativa entre prevalencia parasitaria y los factores sexo y edad en porcinos $(\mathrm{p}<0.05)$.

El factor de riesgo que la población no puede controlar es el factor económico que afecta al $100 \%$ de la población y esa es la condición que pone en mayor riesgo que las personas contraer la teniasis humana.

El $61 \%$ de los pobladores encuestados menciona que los factores sociopolíticos en el Centro Poblado Menor de Bagazán son buenos y el 39\% manifiesta que es regular, en cuanto a los factores económicos el 100\% de los pobladores manifiestan que las condiciones económicas son malas, puesto que todos los habitantes son agricultores con producción tradicional.

El $64 \%$ de los criadores de cerdos mencionan que crían los cerdos sueltos encerrados en un corral, el $24 \%$ crían sueltos sin control, el $6 \%$ lo sueltan por horas y el $4 \%$ crían a los cerdos amarrados. Respecto al sistema de faenamiento el 93\% de los encuestados manifiestan que es regular, el $5 \%$ consideran que es bueno y el $2 \%$ que es malo. 
De $42(100 \%)$ cerdos sacrificados $3(7 \%)$ cerdos estaban infestados con cisticercos en el músculo del lomo la cara, pero era una infestación leve. Este resultado demuestra que en el Centro Poblado Menor de Bagazán existen cerdos infestados con cisticercosis.

El $100 \%$ de las personas en las que se realizó análisis coparasitológico por la técnica cuantitativa de Mc Master modificado y cualitativa de flotación en el laboratorio para detectar teniasis en humanos dieron como resultado negativo.

La propuesta está basada en un sistema de crianza de cerdos sueltos en un corral, donde se les provea las condiciones necesarias de alimentación sana, luego con un sistema de faenamiento adecuado, cuidando de no contaminar en cada etapa con prácticas adecuadas de limpieza durante el faenamiento y un correcto proceso de cocción, permitirán que se disminuya o elimine la presencia de cisticercosis porcina.

\section{Referencias bibliográficas}

1. World Health Organization. WHO/FAO/OIE Guidelines for the surveillance, prevention and control of taeniosis/cysticercosis [Internet]. Murrell KD, editor. 2005. Disponible en: https://apps.who.int/iris/handle/10665/43291

2. Engels D, Urbani C, Belotto A, Meslin F, Savioli L. The control of human (neuro)cysticercosis: which way forward? Acta Trop. junio de 2003;87(1):177-82. doi:https://doi.org/10.1016/S0001-706X(03)00064-0

3. The Cysticercosis Working Group. The marketing of cysticercotic pigs in the Sierra of Peru. Bull World Health Organ [Internet]. 1993;71(2):223-8. Disponible en: https://apps.who.int/iris/handle/10665/47798

4. Castro V. Prevalencia de cisticercosis porcina, comparación de examen de lengua y ensayo de electroinmuno transferencia blot en Maceda, Tarapoto - departamento de San Martín. Universidad Nacional Mayor de San Marcos; 1991.

5. Garcia HH, Gonzalez AE, Rodriguez S, Gonzalvez G, Llanos-Zavalaga F, Tsang VC., et al. Epidemiología y control de la cisticercosis en el Perú. Rev Peru Med Exp Salud Publica. 23 de diciembre de 2010;27(4):592-7. doi:https://doi.org/10.17843/rpmesp.2010.274.1533

6. Toledo RCC, Franco JB, Freitas LS, Katielli C, Freitas ARF de. Complexo teníase / cisticercose: uma revisão. Hig Aliment [Internet]. 2018;32(282-283):30-4. Disponible en: https://docs.bvsalud.org/biblioref/2018/10/916509/282-283-jul-ago-2018-30-34.pdf

7. Pinto PSA, Santos WLM, Laerte PA, Acevedo-Nieto EC, Santos TO, Duarte CTD. Perfil epidemiológico da cisticercose bovina e suína em três regiões do estado de Minas Gerais, Brasil. Arq Bras Med Veterinária e Zootec. febrero de 2019;71(1):167-76. doi:https://doi.org/10.1590/1678-4162-10235 
8. Mena A. C, González Z. A, Falcón P. N, Bernal R. T, Ayvar P. V. Incidencia de cisticercosis porcina en el distrito de matapalo, tumbes. Rev Investig Vet del Perú. 15 de marzo de 2013;15(1):63-9. doi:https://doi.org/10.15381/rivep.v15i1.1564

9. Palomino Iturrarán JG. Prevalencia de cisticercosis porcina por inspección en el camal municipal del cantón Huaquillas provincia de El Oro [Internet]. Universidad Técnica Machala; 2014. Disponible en: http://repositorio.utmachala.edu.ec/handle/48000/1471

10. Mori Raygada CA. Incidencia de cisticercosis porcina en el camal municipal del distrito de Yurimaguas en los años 2012-2013 [Internet]. Universidad Nacional de la Amazonía Peruana; 2018. Disponible en: http://repositorio.unapiquitos.edu.pe/handle/20.500.12737/5467

11. Lightowlers MW, Garcia HH, Gauci CG, Donadeu M. Monitoring the outcomes of interventions against Taenia solium : options and suggestions. Parasite Immunol. 2016;38:158-69. doi:https://doi.org/10.1111/pim.12291

12. de Arruda Pinto PS. Inspeção e higiene de carnes. Segunda Ed. UFV, editor. 2014.

\section{Conflicto de intereses}

En el presente trabajo de investigación, no existe un potencial conflicto de intereses.

\section{Contribuciones de los autores}

Hugo Sánchez-Cárdenas: Muestreo y recolección de muestras coprológicas, coordinación con las autoridades y criadores de porcinos. Contribución en la redacción del artículo.

Víctor Puicón: Procesamiento de muestras coprológicas y contribución en la redacción del artículo.

Heriberto Arévalo-Rámirez: Elaboración del diseño de la investigación, contribución con el análisis estadístico. Contribución en la redacción del artículo. 